\title{
Representational difference analysis of CDNA for the detection of differential gene expression in bacteria: development using a model of iron-regulated gene expression in Neisseria meningitidis
}

\author{
Lucas D. Bowler, ${ }^{1}$ Mike Hubank ${ }^{2}$ and Brian G. Spratt ${ }^{1,3}$ \\ Author for correspondence: Lucas D. Bowler. Tel: +44 1273 678309. Fax: +44 1273678433. \\ e-mail: bafn7@sussex.ac.uk
}

1 School of Biological Sciences, University of Sussex, Brighton BN1 9QG, UK

2 Trafford Centre for Medical Research, University of Sussex, Brighton BN1 9RY, UK

3 Wellcome Trust Centre for the Epidemiology of Infectious Disease, Department of Zoology, University of Oxford Oxford OX1 3PS, UK

\begin{abstract}
Representational difference analysis of CDNA (CDNA RDA) provides a powerful technique for the identification of specific differences between two mRNA populations. The method has previously been used to analyse differential gene expression in eukaryotes, but until now has not been successfully applied to prokaryotes. A strain of Neisseria meningitidis with a deletion of the ironregulated lactoferrin-binding protein A (IbpA) gene, grown under iron-replete conditions, and the isogenic parent strain, grown under iron limitation, were used as a model for developing CDNA RDA for use with bacteria. In this system, the technique should specifically detect the differential expression of the IbpA gene in the parent strain, along with other genes whose expression is switched on (or up-regulated) under iron-deficient conditions. Since cDNA RDA requires high-quality, representative mRNA, a variety of methods for the isolation of RNA were evaluated. A triisopropylnaphthalene sulphonic acid/ $p$-aminosalicylic acid-based technique was found to give the best results. CDNA was prepared from total RNA isolated from the two $N$. meningitidis strains and subjected to an adapted CDNA RDA procedure. The method resulted in the amplification of five major PCR products, which included fragments of the IbpA gene and the iron-regulated RTX-like toxin gene (frpC), thus validating the technique for use with bacteria.
\end{abstract}

Keywords: cDNA representational difference analysis, differential gene expression, RNA isolation, iron regulation, Neisseria meningitidis

\section{INTRODUCTION}

Pathogenic bacteria typically encounter multiple microenvironments during the infection process and must interact with many different types of host cells. The infecting bacterium therefore has to recognize and adapt to these changing conditions in order to survive. Successful pathogens have accordingly evolved a variety of specific gene products that facilitate their survival and growth within the host, as well as mechanisms to regulate expression of these virulence-associated genes in response to their surroundings. Typically, recognition of various environmental cues results in the co-ordinate

Abbreviations: CDNA RDA, representational difference analysis of CDNA; PAS, $p$-aminosalicylic acid; TNS, triisopropylnaphthalene sulphonic acid. regulation of multiple virulence-associated genes, ensuring that genes required at specific stages of the disease process are expressed only at the appropriate times (Miller et al., 1989; Mekalanos, 1992).

Identifying such differential gene expression is a frequent goal in modern biomedical research, and as a consequence, a variety of differential screening methods have been developed over the last few years with varying degrees of success (Soares, 1997; Sagerström et al., 1997). Many of these techniques require large quantities of mRNA and are relatively insensitive, although a few take advantage of PCR to amplify messages to detectable levels. These include differential display PCR (DDPCR), the conceptually similar technique, RNA fingerprinting by arbitrarily primed PCR (AP-PCR), and representational difference analysis of cDNA (cDNA 
RDA). cDNA RDA is a cDNA-specific modification of the PCR-based subtractive hybridization technique of representational difference analysis first described for application to whole genomes by Lisitsyn et al. (1993). cDNA RDA employs a positive selection approach in which target cDNA fragments are sequentially enriched by favourable hybridization kinetics and subsequently amplified by PCR. In contrast to DD- or AP-PCR, cDNA RDA has the major advantage that sequences common to both groups are eliminated, which greatly simplifies the interpretation of results and identification of the differentially expressed genes. In addition, the exponential degree of enrichment achieved by the use of PCR in cDNA RDA enables the detection of very rare transcripts (Hubank \& Schatz, 1999).

A number of microarray-based methods for the detection of differentially expressed genes have been described recently (De Saizieu et al., 1998; Ramsay, 1998). These involve the gridding of PCR products or oligonucleotides corresponding to all of the genes of the organism under study onto a solid support so that the levels of expression of each gene under any growth condition can be monitored by hybridization with labelled RNA preparations. Microarray technology has the potential to revolutionize the study of differential gene expression but there are currently a number of drawbacks to this approach. Firstly, application of the methodology is limited to organisms whose genomes have been sequenced. Secondly, although the method has been recently used successfully for bacterial transcript imaging (De Saizieu et al., 1998), it does not at present have the sensitivity of PCR-based techniques such as cDNA RDA.

Given these considerations, it is apparent that cDNA RDA, with its wide applicability and high sensitivity, is potentially one of the most powerful methods for the study of mechanisms of microbial pathogenesis currently available. The cDNA RDA technique is particularly useful as, in principle, it can in the same experiment analyse differences in gene expression in both the pathogen and host during different stages of their interaction. Furthermore, the sensitivity and specificity of the method should allow total (host plus pathogen) RNA preparations to be used, facilitating the rapid processing of samples without the need for the pathogen and host cells to be separated.

However, whilst the cDNA RDA method is proven with eukaryotic material (Hubank \& Schatz, 1999), the use of cDNA RDA has yet to be reported with bacteria. This is primarily because cDNA RDA requires the isolation of high-quality representative mRNA on a reproducible basis from the organisms under study. This is particularly difficult with prokaryotes since bacterial messages can be very unstable, with mean half-lives of between 1.5 and $2.5 \mathrm{~min}$ at $37^{\circ} \mathrm{C}$ (Alifano et al., 1994), far less than that of eukaryotic mRNAs. Standard purification techniques using oligo(dT) affinity matrices are inapplicable to prokaryotes since bacterial mRNA effectively lack 3'-poly(A) tails, necessitating the use of total RNA preparations, in which most of the RNA will be ribosomal (rRNA). Bacterial cells are also rich in endogenous highly stable ribonucleases which are unaffected by the more commonly used proprietary RNase inhibitors. In this paper, we report development of the cDNA RDA system using total RNA preparations for the identification of differentially expressed genes in bacteria, and its successful application in a model system designed to identify iron-regulated genes in Neisseria meningitidis.

\section{METHODS}

Bacterial cultures. N. meningitidis isolates M986 (B:2a:P1.2) and CE1402 (M986 $\Delta l b p A B$ ) were kindly provided by $\mathrm{Dr}$ Annika Pettersson (University of Utrecht, The Netherlands). All strains were cultured, with shaking, in Mueller-Hinton $(\mathrm{MH})$ broth at $37^{\circ} \mathrm{C}$. Optimal induction of iron-regulated gene expression was achieved by the addition of $25 \mathrm{mM}$ desferrioxamine to the growth medium. MH broth for ironsufficient culture was supplemented with $150 \mu \mathrm{M} \mathrm{Fe}\left(\mathrm{NO}_{3}\right)_{3}$. Overnight cultures of the $N$. meningitidis strains in unsupplemented media were used to inoculate MH broth (1:100) for growth under iron-limited (M986) or iron-sufficient (CE1402) conditions. Cells were grown for $4 \mathrm{~h}$ (to approx. mid-exponential phase, as determined by growth rate analyses) for RNA isolation.

RNA preparation. Total RNA was isolated by a procedure adapted from the triisopropylnaphthalene sulphonic acid (TNS)/p-aminosalicylic acid (PAS) method of Felipe et al. (1993). Work was carried out on ice where possible. Water was RNase-free, UHP quality (ELGA) and was monitored for RNase contamination using the RNaseAlert kit (Ambion). Bacterial cultures were harvested by centrifugation of $10 \mathrm{ml}$ samples in Falcon tubes at 6000 r.p.m. for 5 min at $1{ }^{\circ} \mathrm{C}$. Cell pellets were quickly resuspended in $1.5 \mathrm{ml}$ cold fresh TNS/ PAS buffer containing $2 \%$ TNS (Phase Separation), $6 \%$ PAS (Sigma), 1\% SDS, $50 \mathrm{mM}$ EDTA, $250 \mathrm{mM} \mathrm{NaCl}, 200 \mathrm{mM}$ PIPES $\mathrm{pH} 6 \cdot 0,6 \%$ n-butanol, and $100 \mathrm{mM}$ 2-mercaptoethanol. Acid phenol/chloroform (5:1, v/v, acid equilibrated to $\mathrm{pH} 4 \cdot 7,1.5 \mathrm{ml})$ and chloroform/isoamyl alcohol $(49: 1, \mathrm{v} / \mathrm{v}$, $300 \mu \mathrm{l})$ were added, and the mixture was vortex-mixed for $3 \times 10 \mathrm{~s}$. Samples were spun at $16000 \mathrm{~g}$ for $5 \mathrm{~min}$ at $1{ }^{\circ} \mathrm{C}$ and the aqueous phase carefully transferred to new tubes. The aqueous phase was re-extracted with 1 vol. acid phenol/ chloroform plus $0 \cdot 2$ vol. chloroform/isoamyl alcohol, and again with 1 vol. chloroform. RNA was precipitated by incubation with $2.5 \mathrm{M} \mathrm{LiCl}, 25 \mathrm{mM}$ EDTA, $\mathrm{pH} 8.0$ for at least $2 \mathrm{~h}$ at $-20{ }^{\circ} \mathrm{C}$. Pellets were washed twice with $70 \%$ ethanol and resuspended to $1 \mathrm{mg} \mathrm{ml}^{-1}$ in $3 \mathrm{mM} \mathrm{CaCl}_{2}, 6 \mathrm{mM} \mathrm{MgCl}_{2}$, $50 \mathrm{mM}$ TES pH 7.5, plus 500 U RNase block $\mathrm{ml}^{-1}$ (Stratagene). Contaminating DNA was removed by incubation with RNasefree DNase I (Ambion) for $45 \mathrm{~min}$ at $37^{\circ} \mathrm{C}$. The reaction was terminated by extraction with 1 vol. acid phenol followed by a further extraction with 1 vol. chloroform. RNA was then precipitated with $0 \cdot 2$ vol. $10 \mathrm{M}$ ammonium acetate and 1 vol. 2-propanol for at least $2 \mathrm{~h}$ at $-20^{\circ} \mathrm{C}$. Pellets were washed twice with $70 \%$ ethanol and resuspended to $1 \mathrm{mg} \mathrm{ml}^{-1}$ in water containing $0.1 \mathrm{mM}$ EDTA plus $600 \mathrm{U}$ RNase block $\mathrm{ml}^{-1}$. Quantification and crude quality assessment were done by measuring optical density of the preparation at $260 \mathrm{~nm}$ and $280 \mathrm{~nm}$, and by examination on non-denaturing agarose gels $(1 \%)$ in Tris/borate/EDTA (TBE) buffer $\mathrm{pH} 7.8$ (this was found to give better, more consistent results with RNA than the usual TBE $\mathrm{pH}$ 8.4) stained with ethidium bromide. Aliquots of the total RNA preparations were stored at $-80^{\circ} \mathrm{C}$ until use. 
cDNA synthesis. cDNA synthesis was performed by randompriming of total RNA using the Pharmacia TimeSaver cDNA synthesis kit. The protocol was according to the manufacturer's instructions except, after $20 \mathrm{~min}$ incubation of the first-strand reaction at $37^{\circ} \mathrm{C}, 1 \mu \mathrm{l}$ Superscript II reverse transcriptase (Stratagene) was added to the reaction mix and incubation was continued for a further $1 \mathrm{~h}$. The second-strand incubation was at $12^{\circ} \mathrm{C}$ for $1 \mathrm{~h}$, and then $22^{\circ} \mathrm{C}$ for a further $2 \mathrm{~h}$.

Isolation of $\boldsymbol{N}$. meningitidis rRNA genes and generation of rRNA representations. Synthetic oligonucleotide primers, based on published meningococcal rRNA sequences, were used to amplify the $16 \mathrm{~S}$ and $23 \mathrm{~S}$ rRNA genes from $N$. meningitidis M986 chromosomal DNA isolated using the Genomic-Tip 100/G system (Qiagen). The primer pairs used were as follows: 16S rRNA, 16Sup (5'-CATAAGAGTTTGATCCTGGCT-3') and 16Sdn (5'-GTCATGAAGCATACCGTGGT-3'); 23S rRNA, 23Sup (5'-CAGGT/CGGATGCCTTGGCGA-3') and 23Sdn (5'-AGAGTCAAGCCTCACGA/GGCA-3'). The rRNA gene products were digested with DpnII and the R-Bgl-12/24 adapters ligated as described by Hubank \& Schatz (1999) for the generation of cDNA RDA representations from cDNA. Eighteen cycles of PCR were used to produce the neisserial rRNA representations.

CDNA RDA. The basic method used was as described by Hubank \& Schatz (1994), but with the following modifications. HPLC-purified oligonucleotides were used throughout. Driver and tester components were generated by PCR using 18 cycles of amplification of the R-Bgl-12/24 adapterligated, DpnII-restricted, cDNA (R-Bgl-12, 5'-GATCTGCGGTGA-3'; R-Bgl-24, 5'-AGCACTCTCCAGCCTCTCACCGCA-3'). Digested/unligated oligonucleotide linkers (-Bgl$12 / 24$ series) were separated from the tester when necessary using QIAquick Spin columns (Qiagen). The driver used comprised $50 \%$ N. meningitidis CE1402-derived representation and $25 \%$ each of representations derived from the $16 \mathrm{~S}$ and 23S rRNA genes of N. meningitidis M986. Four rounds of subtractive hybridization and selective hybridization were used. The tester: driver ratios were 1:100 [difference product (DP) 1]; 1:800 (DP2); 1:5000 (DP3). A full protocol can be supplied on request.

Cloning and sequencing of difference products. Final difference products were excised from agarose gels, cloned into the pGEM-T vector (Promega) and sequenced in both directions using M13 $(-21)$ and M13 reverse primers and dRhodamine Terminator chemistry on an ABI 377 DNA sequencer. Resulting sequences were compared to the GenBank database and to the N. meningitidis (Sanger Centre, Cambridge, UK, and The Institute for Genome Research, Rockville, MD, USA) and Neisseria gonorrhoeae (University of Oklahoma, OK, USA) databases using the BLAST program (Altschul et al., 1990).

Southern blotting. Representations were electrophoresed through $1 \%$ non-denaturing agarose gels in TBE buffer and transferred to nylon membrane (Hybond-N, Amersham), using the downward alkaline blotting method of Chomczynski (1992). DNA probes were produced by PCR amplification using pairs of synthetic oligonucleotide primers directed to the $5^{\prime}$ and $3^{\prime}$ ends of the identified difference products. Nonradioactive labelling and detection were carried out using the Gene Images (Amersham) labelling and detection kit according to the manufacturer's instructions.

RT-PCR. All RNA preparations were pre-screened for residual DNA contamination by PCR using the primer pairs synthesized for the Southern blotting analysis. To confirm difference products as genuine, RT-PCR was performed using the Pharmacia Ready-To-Go RT-PCR bead system. One-step reactions $(0.5 \mathrm{ml})$ were carried out using $200 \mathrm{ng}$ total RNA for each reaction and the same primer pairs. To compare relative levels of expression, RT-PCR was carried out using an internal control to normalize the samples. The control used was the house-keeping gene $g \ln A$, whose expression is not ironregulated. The primer pair for this purpose was GlnAup1165 (5'-CCCGGATGGATTTTGTTTTG-3') and GlnAdn600 (5'CACAAAACCCGTTACGAAATCA-3'), which amplify a PCR product of approximately $750 \mathrm{bp}$. First-strand synthesis was carried out at $46^{\circ} \mathrm{C}$ for $30 \mathrm{~min}$. The semi-quantitative PCR amplification of the cDNA first strand was performed for 17 cycles.

\section{RESULTS}

\section{Isolation of high-quality RNA}

Successful development of the cDNA RDA technique requires the reproducible isolation of very high-quality representative mRNA preparations from bacteria. A major part of this study accordingly concerned the development of the optimum method for the preparation of RNA from N. meningitidis. We critically evaluated a number of different isolation protocols and, given the instability of bacterial mRNA, emphasis was placed on those approaches which lysed cells rapidly. Ultimately, the selected protocols were progressively modified to give the best results with $N$. meningitidis.

Methods based on the homogenization of cellular material in guanidine. $\mathrm{HCl}$ or hot phenol have often proven to be unreliable (Groppe \& Morse, 1993), and this was also found to be the case in this study. These

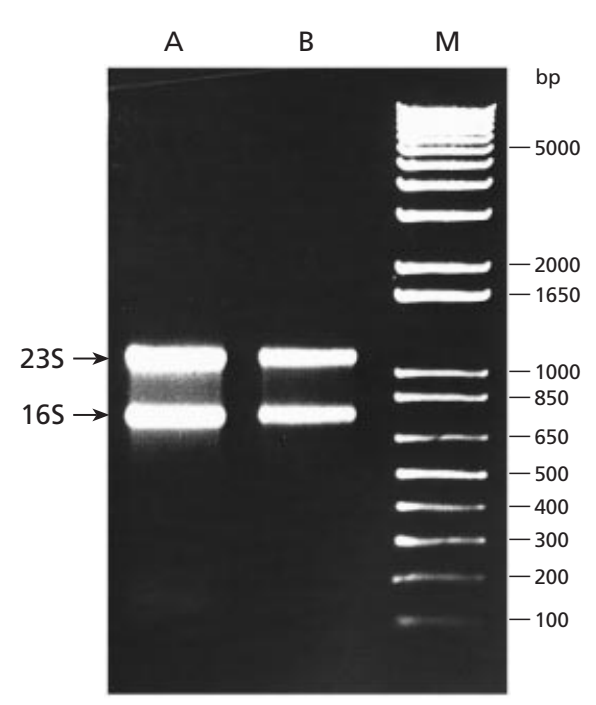

Fig. 1. Analysis of $N$. meningitidis RNA. Total RNA was prepared from $N$. meningitidis using the TNS/PAS method at $\mathrm{pH}$ 6.0 and was fractionated on a $1 \%$ non-denaturing agarose gel. Lanes: A, total RNA isolated from strain CE1402 grown under iron-sufficient conditions; $B$, total RNA isolated from strain M986 grown under iron limitation; $M$, molecular mass markers. Positions of the dominant $16 \mathrm{~S}$ and 235 rRNA bands are indicated by arrows. 


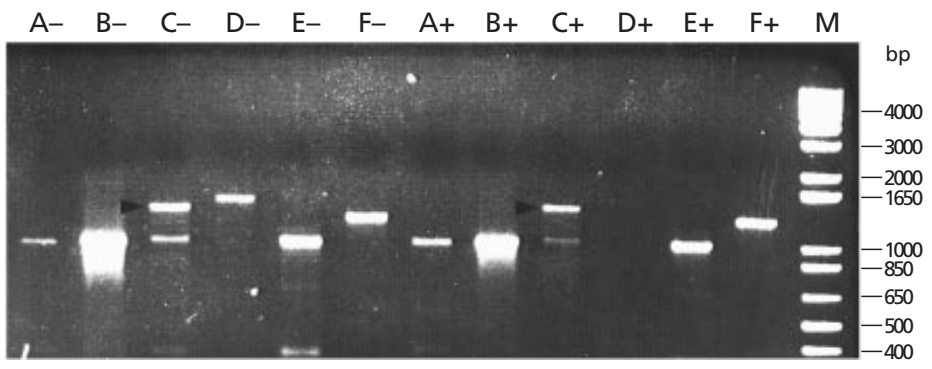

Fig. 2. Detection of gene transcripts by RTPCR (non-quantitative). RT-PCR was carried out using total RNA produced by the TNS/PAS method at $\mathrm{pH} 6.0$ and primers specific for the $N$. meningitidis aroE (lanes $\mathrm{A}-, \mathrm{A}+$ ); fbpA (lanes $\mathrm{B}-, \mathrm{B}+$ ); $g \ln A$ (lanes $\mathrm{C}_{-}, \mathrm{C}_{+}$; relevant product is indicated by arrows); IbpA (lanes $\mathrm{D}-, \mathrm{D}+$ ); penA (lanes $\mathrm{E}-, \mathrm{E}+$ ); and rho-transcription terminator $(\rho)$ (lanes $\mathbf{F}-, \mathbf{F}+$ ) genes. Molecular size markers (in lane $\mathrm{M}$ ) are indicated. Total RNA was from $N$. meningitidis M986 grown under iron limitation (lanes $\mathrm{A}-$ to $\mathrm{F}-$ inclusive) or $N$. meningitidis CE1402 grown under iron-sufficient conditions (lanes $\mathrm{A}+$ to $\mathrm{F}+$ inclusive).

techniques were therefore not considered further. However, relatively rapid and efficient techniques involving the use of more powerful chaotropic agents such as guanidine isothiocyanate (GITC) have been developed (Chirgwin et al., 1979; Chomczynski \& Sacchi, 1987). Novel approaches using detergents such as Catrimox-14 (Dahle \& Macfarlane, 1993), TNS (Felipe et al., 1993) and cetyltrimethylammonium bromide (CTAB) (Cheung et al., 1994) have also been described, and apparently used for the production of high-quality, high-purity, intact mRNA. All of these newer methods were assessed for their ability to provide high-quality RNA from N. meningitidis. Published methods based on the use of GITC differ widely in the $\mathrm{pH}$ at which the lysis/extraction solutions are buffered (in the range $\mathrm{pH}$ $5 \cdot 0-7 \cdot 5)$. As it has been reported that bacterial RNA may be more stable at lower $\mathrm{pH}$ (Noonberg, 1995), all isolation protocols were modified by the substitution of a variety of alternative buffering systems.

RNA derived using these methods was initially assessed qualitatively by non-denaturing agarose gel electrophoresis. Good-quality preparations were characterized by the presence of the dominant $16 \mathrm{~S}$ and $23 \mathrm{~S}$ rRNA species appearing as fairly sharp intense bands with apparent sizes of about $750 \mathrm{bp}$ and just over $1000 \mathrm{bp}$, respectively, relative to double-stranded DNA molecular size markers (Fig. 1). In addition, a smaller more diffuse band comprising low-molecular-mass RNA should also be visible. Some diffuse smearing may be visible between $16 \mathrm{~S}$ and $23 \mathrm{~S}$ bands and a little below the $16 \mathrm{~S}$ band, but there should be a clear region between this and the low-molecular-mass RNA band towards the bottom of the gel. Using these criteria, RNA isolation at $\mathrm{pH} 4 \cdot 0$, by whatever method, resulted in considerable degradation of the nucleic acid and a $\mathrm{pH} \geqslant 8.0$ also led to some degradation. Most of the various methods of isolation we used appeared to have $\mathrm{pH}$ optima of $\mathrm{pH}$ 5.5-6.5 and the yields from each were broadly comparable, although RNA preparations using Catrimox-14 were less efficient and those using GITC were slightly more efficient (data not shown).

This non-denaturing gel analysis provided a good overall assessment of RNA quality, but provided no information concerning how representative the mRNA component of each preparation was, or any idea of its integrity. Accordingly, Northern blot analyses were carried out on RNA isolated from bacteria grown under iron-limited conditions, each RNA preparation being screened using a variety of chemiluminescently labelled DNA probes, derived using PCR and selected to identify meningococcal transcripts of varying abundance.

This approach indicated that the RNA isolation method of Chomczynski \& Sacchi (1987) and the TNS/ PAS protocol (Felipe et al., 1993) carried out at $\mathrm{pH} 6.0$ or $\mathrm{pH}$ $7 \cdot 0$ were the most promising. However, given the low sensitivity of Northern blot analysis, RT-PCR was used as another assay for the presence of mRNAs from a variety of genes. The transcripts targeted were of the iron-regulated lactoferrin-binding protein A $(l b p A)$ (Pettersson et al., 1994) and periplasmic iron-binding protein $(f b p A)$ (Berish et al., 1990) genes, both expressed at relatively high levels under iron limitation; the shikimate dehydrogenase (aroE) and glutamine synthetase $(g \ln A)$ genes (Zhou et al., 1997), expressed at medium levels; and the penicillin-binding protein 2 (penA) (Spratt et al., 1989) and rho transcription terminator (Opperman \& Richardson, 1994) genes, both expressed at relatively low levels.

Multiple total RNA preparations derived using each isolation method were tested by RT-PCR to check reproducibility and the integrity of the RNA transcripts. RNA prepared from N. meningitidis M986 using the TNS/PAS method at $\mathrm{pH} 6.0$, with the addition of $n$ butanol to prevent PAS precipitation at low temperatures, was selected as the most suitable for use as the template for cDNA synthesis for cDNA RDA. Fig. 2 shows the results from RT-PCR screening of a total RNA preparation derived using the modified TNS/PAS method.

\section{Preparation of CDNA from total RNA preparations}

Bacterial cDNA RDA requires the production of highquality representative cDNA from total RNA preparations. Given that about $85 \%$ of the total RNA may be rRNA (Farrell, 1993), it was initially thought that the 
(a)

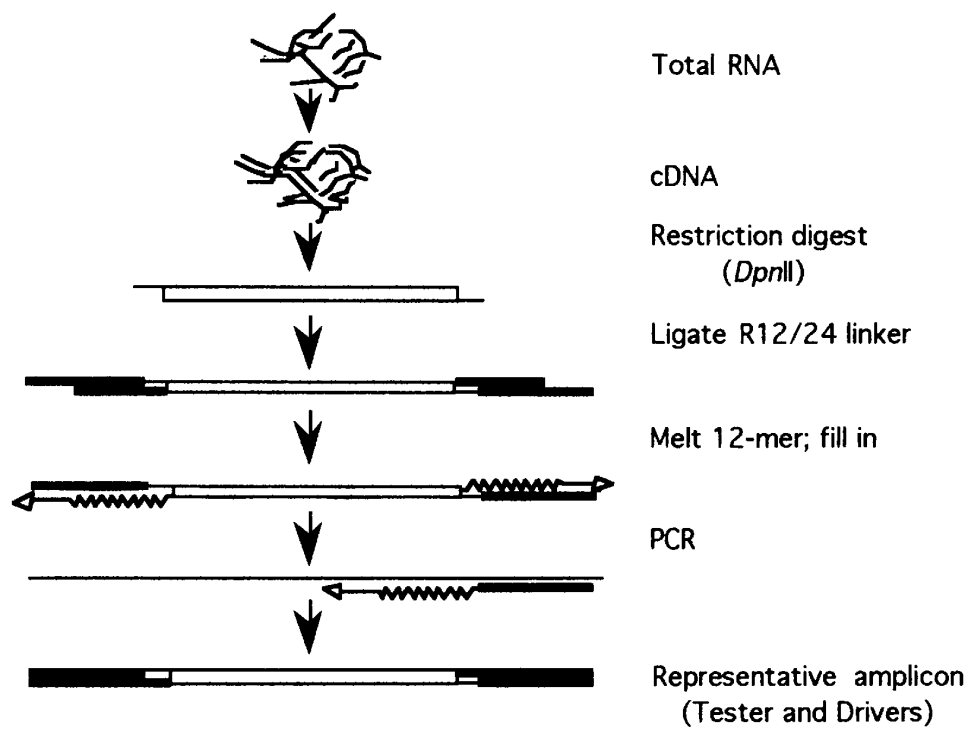

(b)

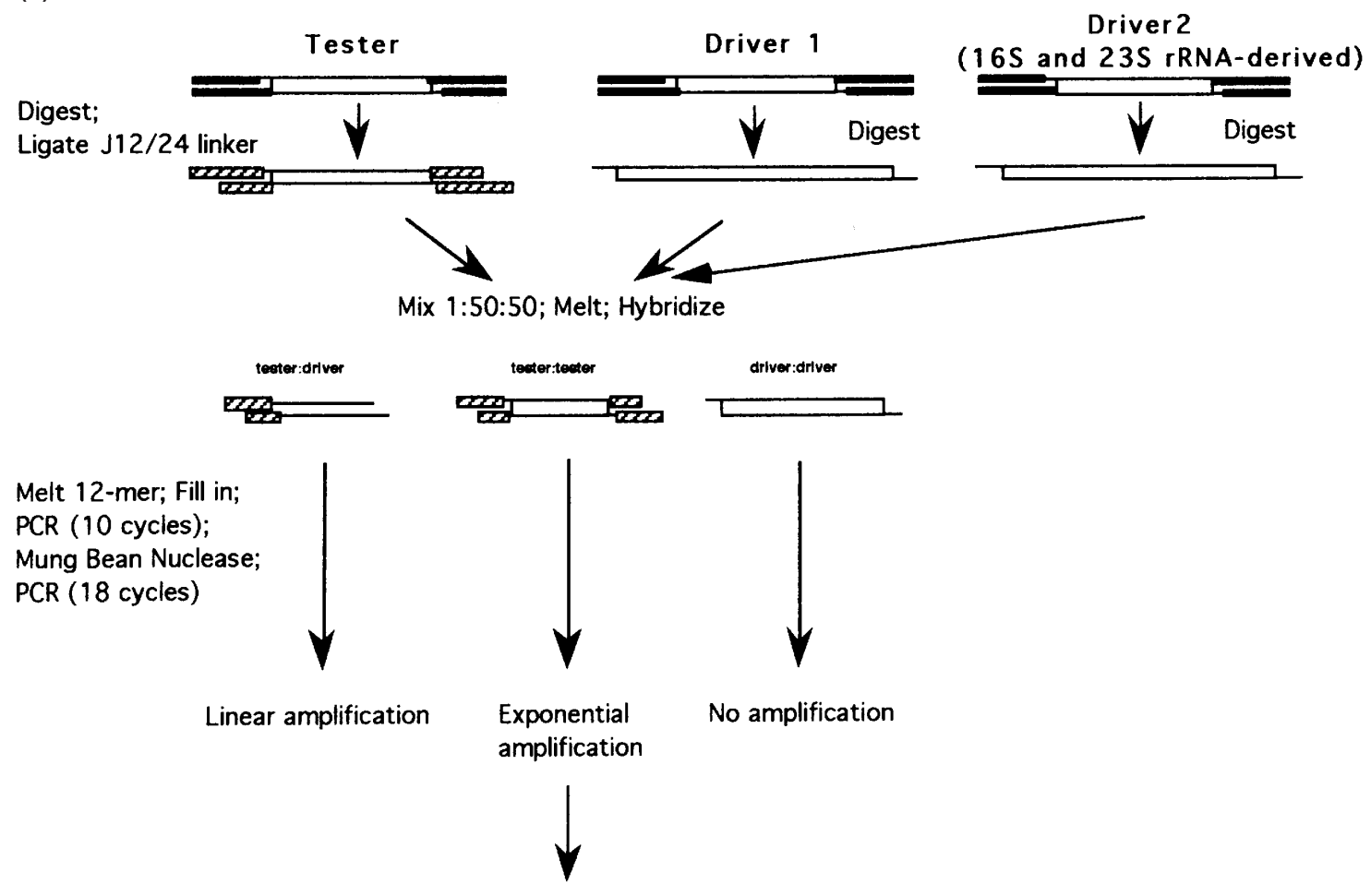

FIRST DIFFERENCE PRODUCT

Fig. 3. Schematic representation of the CDNA RDA procedure to the first difference product. Solid boxes represent the Roligonucleotides used to generate the representations. Cross-hatched boxes show the J- (or N-) oligonucleotides used to generate difference products. (a) Generation of tester and driver components. Zig-zag arrows indicate DNA synthesis. (b) cDNA RDA. To generate second and third difference products, products are reintroduced into the scheme at the tester stage in the proportions indicated in Methods. To increase selection against the amplification of rRNA-derived cDNA fragments, the normal driver (driver 1 ) is supplemented with additional rRNA sequences (driver 2). To generate driver 2 , N. meningitidis CE1402 $16 \mathrm{~S}$ and $23 \mathrm{~S}$ genes isolated by PCR were substituted for CDNA. 
total RNA preparations would have to be depleted of these components as the large excess of cDNA derived from rRNA could interfere with the cDNA RDA procedure, particularly during the subtractive and PCR enrichment steps. Standard mRNA purification techniques using poly $(\mathrm{T})$ affinity matrices were unlikely to be very useful since prokaryotic messages appear to be only poorly polyadenylated (Manley, 1995). Furthermore, little is known concerning the proportion of mRNAs which do possess short poly(A) tails. A variety of methods have been described which use antisense probes to remove unwanted sequences from complex cDNA mixtures (Duguid et al., 1988; Swaroop et al., 1991; Sagerström et al., 1997; Shepard \& Rae, 1997), and at least one report describing the subtraction of rRNA from a total RNA preparation has been published (Plum \& Clark-Curtiss, 1994). Numerous attempts were made to deplete the total RNA preparations of rRNA with these types of approach. However, we found that subtraction of rRNA using these methods was very inefficient. Moreover, Northern blot analysis showed that repeated subtractions to obtain more complete depletion of rRNA resulted in the loss of some mRNAs. The cDNA RDA technique was therefore developed using N. meningitidis total RNA preparations, and modifications introduced into the protocol which increased the direct selection against amplification of rRNA sequences.

\section{The cDNA RDA procedure}

Examination of differential gene expression using cDNA RDA requires the sampling of a population of cells grown under the condition(s) of interest (in this study, under iron limitation - the so-called 'tester' component), and a population grown in exactly the same way but differing in the condition(s) of interest (in this case, with iron-sufficiency - the 'driver' component). mRNA is extracted from both cell populations and used as a template for cDNA synthesis. The derived cDNAs are digested with a frequently cutting restriction enzyme. Synthetic oligonucleotides are ligated to the ends of these restriction fragments. PCR with primers specific to these oligonucleotides is then used to amplify each of the DNA fragments in the different populations (Fig. 3a). After amplification, the oligonucleotides are removed with the same restriction enzyme and the amplified cDNA fragments are purified (these preparations are referred to as 'representations'). For the subsequent hybridization and amplification steps, different oligonucleotides are ligated only to the tester DNA fragments. Tester and driver DNAs (the latter in excess) are mixed, denatured and allowed to hybridize. PCR using primers specific to the new oligonucleotide extensions results in exponential amplification of sequences unique to the tester DNA population, whereas sequences present in both populations, or only in the driver population, will either be amplified linearly or not at all (Fig. 3b). By repeating these steps, the degree of enrichment of sequences unique to the tester population is increased, and after several cycles of this procedure a number of discrete PCR products are obtained (the difference products) that represent the mRNAs unique to the tester population. These products can then be cloned and characterized.

To adapt the basic cDNA RDA protocol for use with bacteria, a number of modifications were necessary. Since bacterial mRNA possesses only relatively short or no poly(A) tails, we used random synthetic oligonucleotide hexamers, rather than oligo(dT) to prime cDNA synthesis from total RNA preparations. The derived cDNA was then digested with DpnII. This restriction endonuclease was selected because it gave internal fragments of the N. meningitidis M986 ironregulated $l b p A$ gene which were amplifiable under the competitive PCR conditions of the cDNA RDA, and therefore acted as a positive control. This was necessary since PCR as used in cDNA RDA tends to favour amplification of fragments in the range $\approx 200-600 \mathrm{bp}$. All oligonucleotides used in cDNA RDA were of HPLC purity, which is known to improve specificity in PCR and has been reported to increase the sensitivity of cDNA RDA with eukaryotic material (O'Neill \& Sinclair, 1997). Because of the probable abundance of rRNA-derived sequences and the potential for their interference in the subsequent stages of cDNA RDA, we increased the selection against amplification of this material. Accordingly, additional representations were derived from N. meningitidis M986 $16 \mathrm{~S}$ and 23S rRNA genes and used to supplement the normal RNA-derived driver in the ratio $1: 1: 2$ respectively. The addition of supplementary rRNA-derived representations to the driver component has the additional effect of reducing the stringency of the analysis. We therefore used three cycles of subtractive hybridization and amplification. A tester to driver ratio of 1:5000 was used to generate the third difference product.

Using these modifications (shown schematically in Fig. 3), two independent cDNA RDA experiments were performed. In both, the same pattern of five major distinct PCR products was obtained from the cDNA RDA of $N$. meningitidis, which ideally would represent mRNAs derived from the $l b p A$ gene and from other meningococcal genes that are iron-regulated. The PCR products were cloned and the inserts in a number of the clones sequenced. Of 28 sequences from the first analysis (Fig. 4, lane A), six (21\%) were found to correspond to four different amplifiable fragments of the $l b p A$ gene, and one $(4 \%)$ to a region of the N. meningitidis RTXlike toxin gene $(f r p C)$, which is also known to be ironregulated. Fourteen of the remainder comprised unidentified meningococcal ORFs $(50 \%)$ and seven $(25 \%)$ were rRNA-derived sequences. The five amplified products from the second independent cDNA RDA experiment (Fig. 4, lane B) also included the same four fragments from the $l b p A$ gene and fragment of frpC.

Verification that the cloned difference products corresponded to genes that were differentially expressed or up-regulated in N. meningitidis was carried out in two stages. First, by using a fragment of each of the cloned products as probes on Southern blots of the original 


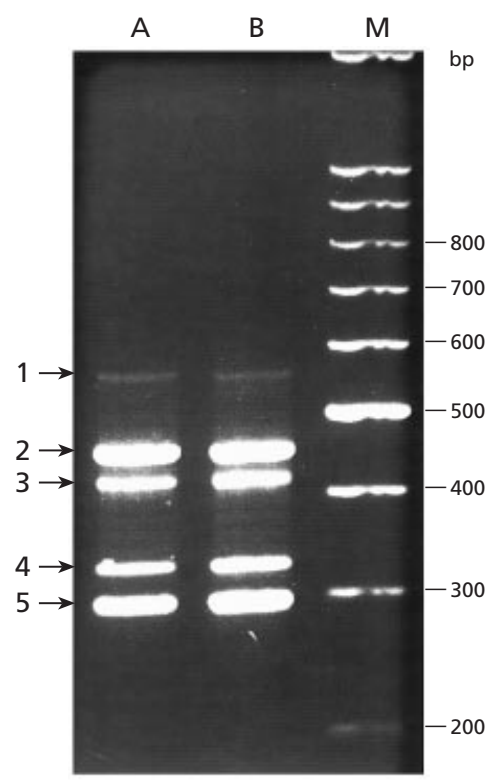

Fig. 4. Analysis of difference products produced by CDNA RDA. CDNA RDA was performed using total RNA prepared from $N$. meningitidis strain M986 grown under iron limitation and strain CE1402 $\triangle / b p A B$ grown under iron-replete conditions. Lanes $A$ and $B$ show the DNA fragments amplified in two independent experiments initiated from separate aliquots of the same total RNA preparations. Lane $M$, molecular mass markers. Arrows indicate the five bands isolated, cloned and sequenced.

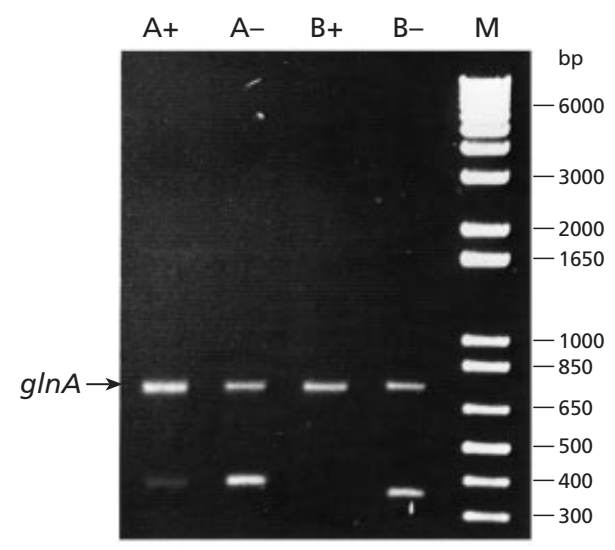

Fig. 5. Expression levels (semi-quantitative) of $I b p A$ and $\operatorname{frpC}$ in the starting RNA populations. RT-PCR was used to monitor the level of expression of the $\operatorname{frpC}$ (lanes $\mathrm{A}_{+}, \mathrm{A}_{-}$) and IbpA (lanes $\mathrm{B}+, \mathrm{B}-$ ) genes in the total RNA preparations used for CDNA RDA. Total RNA was from strain CE1402 $\triangle$ lbpAB grown under iron-replete conditions (lanes $A+, B+$ ) or from strain M986 grown under iron limitation (lanes $\mathrm{A}-, \mathrm{B}-$ ). glnA was used as an internal control gene whose level of expression is independent of iron availability. Lane $M$, molecular mass markers.

cDNA representations, and then by RT-PCR, which was favoured over Northern blot analysis because of its greater sensitivity. RT-PCR was performed using primers designed from the sequences of the difference products. To compare relative levels of expression between samples derived from N. meningitidis grown under iron-restricted or iron-sufficient conditions (i.e. to detect up-regulation rather than absolute differences), a non-iron-regulated internal control $(g \ln A)$ was used to normalize samples. Using this approach, it was demonstrated that $\operatorname{fr} \mathrm{C}$ is up-regulated under iron limitation, whereas $l b p A$ was, as expected, an absolute difference (Fig. 5).

Southern blot analysis of the original tester and driver components and RT-PCR of the initial total RNA preparations were used to confirm that the cloned fragments of the $N$. meningitidis genes were genuinely only expressed, or up-regulated, under conditions of iron limitation. In the first experiment (excluding rRNA sequences), 7 out of $21(33 \%)$ and in the second experiment 17 out of $45(38 \%)$ of the cloned fragments were found to be genuinely iron-regulated.

\section{DISCUSSION}

To develop the cDNA RDA methodology for use with bacteria, we have used a model system comprising a strain of N. meningitidis (CE1402) which has a deletion in the genes encoding the iron-regulated components of the lactoferrin receptor $(\triangle l b p A B)$ and its isogenic $l b p A^{+} B^{+}$parent, $N$. meningitidis M986, grown under iron-replete and iron-limited conditions, respectively. A number of well-characterized Neisseria genes are induced under iron limitation (Berish et al., 1990; Litwin \& Calderwood, 1993; Thompson et al., 1993; Pettersson et al., 1994) and the specific identification of these genes by cDNA RDA would provide an excellent way to validate the technique. CDNA RDA has the ability to detect both relative (greater than three- to fivefold) and absolute differences in expression (Hubank \& Schatz, 1999). The detection of relative differences is favoured by the use of lower stringency, whilst absolute differences should be present at low stringencies but will also survive high-stringency subtractions (stringency in this context refers to driver:tester ratios and not hybridization conditions of salt and temperature, which remain constant). We used an $l b p A B$ deletion mutant to provide a known absolute difference in gene expression, and selected the restriction enzyme (DpnII) to give amplifiable fragments of $l b p A$, in order to simplify the model system. Thus, the identification of regions of $l b p A$ as a genuine difference product was essential in order to validate the method. The identification of other iron-regulated genes whose expression may be upregulated rather than simply switched on, was to be considered a bonus.

In this study we have, as expected, identified several difference products corresponding to regions of $\mathrm{N}$. meningitidis $l b p A$, and in addition detected a fragment of the meningococcal RTX-like toxin gene, $f r p C$, whose expression is known to be up-regulated under conditions of iron limitation (Thompson et al., 1993). Additional iron-regulated genes might have been identified by 
sequencing more inserts from the cloned amplified fragments since individual difference product bands produced by cDNA RDA often comprise many different fragments (M. Hubank, unpublished observation). A second cDNA RDA experiment (Fig. 4, lane B) identified the same parts of the $l b p A$ gene and region of $f_{p} p C$ as genuine difference products, demonstrating the reproducibility of the technique.

It should be emphasized that this paper describes the successful development of the cDNA RDA technique for bacteria, and is not intended to be a thorough analysis of the iron-regulated genes of N. meningitidis. Indeed, most of the previously characterized neisserial ironregulated genes were not detected (e.g. $l b p B, f r p B, f b p A$ and $t b p A$ etc.). However, this was expected since the kinetics of the PCR-coupled subtractive hybridization in cDNA RDA preferentially enrich for DNA fragments in the 200-600 bp range (as can be seen in Fig. 4). Thus, only those genes whose digestion with the chosen restriction enzyme results in fragments within this size range will amplify efficiently and be detected as difference products. For example, from published sequence data, we know that complete $D p n$ II digestion of the $l b p B$ gene from $N$. meningitidis M986 will not result in fragments of a suitable size to give amplifiable products using cDNA RDA. Thus, the failure to detect fragments of the $l b p B$ gene, which was deleted together with the $l b p A$ gene in strain CE1402 (Pettersson et. al., 1998), was unsurprising. It is likely that this also explains the failure to find gene fragments from the periplasmic ferric-binding protein gene, $f b p A$, which, although not sequenced from M986, is highly conserved within both meningococci and N. gonorrhoeae (Zhou \& Spratt, 1992). Analysis of the available $f b p A$ sequences indicates that $D p n I I$ digestion of this gene will also not result in amplifiable products under cDNA RDA. None of the other iron-regulated genes, which tend to be highly variable, have been sequenced from strain M986, which makes it difficult to assess whether they should have been recovered using DpnII. However, examination of the published meningococcal genome sequence shows that $D p n I I$ would cut on average every $600 \mathrm{bp}$ or so, the upper limit for cDNA RDA, suggesting that this enzyme is not the most appropriate for optimal recovery of difference products with $N$. meningitidis. As a consequence of the size constraints on the DNA fragments that can be amplified by cDNA RDA, a thorough analysis to detect all of the genes that are differentially expressed under given conditions requires that cDNA RDA be carried out with a number of different frequently cutting restriction endonucleases.

The percentage of genuine difference products was relatively high (about 33\%), but by using different proportions of rRNA representation in the driver, we believe that the level of rRNA contamination could be reduced further. The driver to tester ratios and/or number of subtractive hybridization/amplification cycles used could also be increased if the number of false positives is high, although it should be noted that this could lead to a failure to identify the more rare differences. In this study, no 5S-rRNA-derived sequences were detected. Nevertheless, given their abundance, it may also be useful to include a 5S rRNA component in driver 2 since in other circumstances restriction of 5S-rRNA-derived cDNA may result in amplifiable fragments. However, the use of an initial additional subtractive step using only rRNA-gene-derived driver was not successful and resulted in the identification of no genuine differences in gene expression. This may be because, although it resulted in increased selection against amplification of rRNA-derived sequences, all other cDNAs were amplified, heavily biasing the resulting cDNA population towards other abundant mRNA sequences common to each population. Subsequent rounds of PCR-coupled subtractive hybridization were then insufficient to isolate genuine difference products. Improved detection of up- or down-regulated gene expression by cDNA RDA can also be obtained using representations which have been depleted of lowcopy sequences by a process known as melt depletion (Hubank \& Schatz, 1994).

The cDNA RDA technique has great potential for studying differential gene expression during hostpathogen interactions during the course of infection, as total RNA prepared from the infected host tissue can be compared to that prepared from host and pathogen grown separately, but under the same environmental conditions. The very high sensitivity and specificity of the cDNA RDA approach should allow the genes switched on, or up- or down-regulated, in both a bacterial pathogen and its host, to be analysed in the same experiment. The demonstration that cDNA RDA can be successfully used with total RNA preparations is therefore important as infected cells can be rapidly processed without the need to separate bacterial cells from those of the host, greatly increasing the chances of identifying differentially expressed genes whose transcripts are short-lived. cDNA RDA should also be of great value in facilitating the application of microarray technology to the study of differential gene expression in bacteria since it could be used to produce probes of higher specificity, enriched for low-abundance signals.

\section{ACKNOWLEDGEMENTS}

This work was supported by the Wellcome Trust. B. G.S. is a Wellcome Trust Principal Research Fellow. We would like to thank Annika Pettersson for providing bacterial strains and advice on iron-regulated gene expression, and Chris Ford, Neil Simpson and Emmanuel Bessent for many useful discussions.

\section{REFERENCES}

Alifano, P., Bruni, C. B. \& Carlomagno, M. S. (1994). Control of mRNA processing and decay in prokaryotes. Genetica 94, 157-172.

Altschul, S. F., Gish, W., Miller, W., Myers, E. W. \& Lipman, D. J. (1990). Basic local alignment search tool. J Mol Biol 215, 403-410.

Berish, S. A., Mietzner, T. A., Mayer, L. W., Genco, C. A., Holloway, B. P. \& Morse, S. A. (1990). Molecular cloning and 
characterisation of the structural gene for the major ironregulated protein expressed by Neisseria gonorrhoeae. J Exp Med 171, 1535-1546.

Cheung, A. L., Eberhardt, K. J. \& Fischetti, V. A. (1994). A method to isolate RNA from gram-positive bacteria and mycobacteria. Anal Biochem 222, 511-514.

Chirgwin, J. M., Przybyla, A. E., MacDonald, R. J. \& Rutter, W. J. (1979). Isolation of biologically active ribonucleic acid from sources enriched in ribonuclease. Biochemistry 18, 5294-5299.

Chomczynski, P. (1992). One-hour downward alkaline capillary transfer for blotting of DNA and RNA. Anal Biochem 201, 134-139.

Chomczynski, P. \& Sacchi, N. (1987). Single-step method of RNA isolation by acid guanidinium thiocyanate-phenol-chloroform extraction. Anal Biochem 162, 156-159.

Dahle, C. E. \& Macfarlane, D. E. (1993). Isolation of RNA from cells in culture using Catrimox-14 cationic surfactant. Biotechniques 15, 1102-1105.

De Saizieu, A., Certa, U., Warrington, J., Gray, C., Keck, W. \& Mous, J. (1998). Bacterial transcript imaging by hybridisation of total RNA to oligonucleotide arrays. Nature Biotechnol 16, $45-48$.

Duguid, J. R., Rohwer, R. G. \& Seed, B. (1988). Isolation of cDNAs of scrapie-modulated RNAs by subtractive hybridisation of a cDNA library. Proc Natl Acad Sci USA 85, 5738-5742.

Farrell, R. E., Jr (1993). RNA Methodologies - a Laboratory Guide for Isolation and Characterisation. San Diego: Academic Press.

Felipe, M. S. S., Rogelin, R., Azevedo, M. O. \& Astolfi-Filho, S. (1993). Extended application of an efficient method for RNA isolation from different organisms. Biotechnol Tech 7, 639-644.

Groppe, J. C. \& Morse, D. E. (1993). Isolation of full-length RNA templates for reverse transcription from tissue rich in RNase and proteoglycans. Anal Biochem 210, 337-343.

Hubank, M. \& Schatz, D. G. (1994). Identifying differences in mRNA expression by representational difference analysis of cDNA. Nucleic Acids Res 22, 5640-5648.

Hubank, M \& Shatz, D. G. (1999). cDNA representational difference analysis: a sensitive and flexible method for the identification of differentially expressed genes. Methods Enzymol 303, 325-349.

Lisitsyn, N., Lisitsyn, N. \& Wigler, M. (1993). Cloning the differences between two complex genomes. Science 259, 946-951.

Litwin, C. M. \& Calderwood, S. B. (1993). Role of iron in regulation of virulence genes. Clin Microbiol Rev 6, 137-149.

Manley, J. L. (1995). Messenger RNA polyadenylation: a universal modification. Proc Natl Acad Sci USA 92, 1800-1801.

Mekalanos, J. J. (1992). Environmental signals controlling expression of virulence determinants in bacteria. J Bacteriol 174, $1-7$.

Miller, J. F., Mekalanos, J. J. \& Falkow, S. (1989). Coordinate regulation and sensory transduction in the control of bacterial virulence. Science 243, 916-922.
Noonberg, S. B. (1995). Effect of pH on RNA degradation during guanidinium extraction. BioTechniques 19, 731-733.

O’Neill, N. J. \& Sinclair, A. H. (1997). Isolation of rare transcripts by representational difference analysis. Nucleic Acids Res 25, 2681-2682.

Opperman, T. \& Richardson, J. P. (1994). Phylogenetic analysis of sequences from diverse bacteria with homology to the Escherichia coli rho gene. J Bacteriol 176, 5033-5043.

Pettersson, A., Klarenbeek, V., Van Deurzen, J., Poolma, J. T. \& Tommassen, J. (1994). Molecular characterization of the structural gene for the lactoferrin receptor of the meningococcal strain H44/76. Microb Pathog 17, 395-408.

Pettersson, A., Prinz, T., Umar, A., van der Biezen, J. \& Tommassen, J. (1998). Molecular characterisation of LbpB, the second lactoferrin-binding protein of Neisseria meningitidis. Mol Microbiol 27, 599-610.

Plum, G. \& Clark-Curtiss, J. E. (1994). Induction of Mycobacterium avium gene expression following phagocytosis by human macrophages. Infect Immun 62, 476-483.

Ramsay, G. (1998). DNA chips: state of the art. Nature Biotechnol $16,40-44$.

Sagerström, C. G., Sun, B. I. \& Sive, H. L. (1997). Subtractive cloning: past, present, and future. Annu Rev Biochem 66, 751-783.

Shepard, A. R. \& Rae, J. L. (1997). Magnetic bead capture of cDNAs from double-stranded plasmid cDNA libraries. Nucleic Acids Res 25, 3183-3185.

Soares, M. B. (1997). Identification and cloning of differentially expressed genes. Curr Opin Biotechnol 8, 542-546.

Spratt, B. G., Zhang, Q.-Y., Jones, D. M., Hutchison, A., Brannigan, J. A. \& Dowson, C. G. (1989). Recruitment of a penicillin-binding protein gene from Neisseria flavescens during the emergence of penicillin resistance in Neisseria meningitidis. Proc Natl Acad Sci USA 86, 8988-8992.

Swaroop, A., Xu, J., Agarwal, N. \& Weissman, S. M. (1991). A simple and efficient cDNA library subtraction procedure: isolation of human retina-specific cDNA clones. Nucleic Acids Res 19, 1954.

Thompson, S. A., Wang, L. \& Sparling, P. F. (1993). Cloning and nucleotide sequence of $f r p C$, a second gene from Neisseria meningitidis encoding a protein similar to RTX cytotoxins. Mol Microbiol 9, 85-96.

Zhou, J. \& Spratt, B. G. (1992). Sequence diversity within the $\arg F$, $f b p$ and recA genes of natural isolates of Neisseria meningitidis: interspecies recombination within the $\operatorname{argF}$ gene. Mol Microbiol 6, 2135-2146.

Zhou, J., Bowler, L. D. \& Spratt, B. G. (1997). Interspecies recombination and phylogenetic distortion within the glutamine synthetase and shikimate dehydrogenase genes of Neisseria meningitidis and commensal Neisseria species. Mol Microbiol 23, 799-812.

Received 7 June 1999; revised 7 September 1999; accepted 9 September 1999. 